\title{
Indikationen zur direkten CT-Venografie
}

Coelho A et al. Usefulness of Direct Computed Tomography Venography in Predicting Inflow for Venous Reconstruction in Chronic Post-thrombotic Syndrome.

Cardiovasc Intervent Radiol 2019; 42: 677-684

Gefäßchirurgen aus Portugal untersuchten, inwieweit bei ausgedehnter chronischer iliofemoraler venöser Obstruktion durch die direkte CT-Venografie (DCTV) Informationen über die Hämodynamik des Hauptzugangs zur V. femoralis communis erhalten werden.

Für ihre retrospektive Untersuchung werteten die Autoren alle DCTVs im Zeitraum von Januar 2014 bis August 2018 aus, die bei symptomatischen Patienten mit iliofemoraler tiefer Venenthrombose durchgeführt worden waren. Dabei wurden nur Patienten eingeschlossen, bei denen eine Rekanalisation möglich war und bei denen vor dem Eingriff eine DCTV und Venografie erfolgte. Je nachdem, ob die $V$. femoralis (FV) oder die V. femoralis profunda (DFV) den dominierenden Einfluss auf die Venografie ausübten, wurden 2 Gruppen definiert, um Prädiktoren für DFV als dominanten Einfluss auf die DCTV zu identifizieren.
Insgesamt konnten 30 DCTVs und nachfolgende Venografien von 30 unterschiedlichen Patientinnen und Patienten überprüft werden. Das Durchschnittsalter der Studiengruppe lag bei 39,9 \pm 12 ,7 Jahren, $80 \%$ waren weiblich. Von den 30 Patientinnen und Patienten hatten $70 \%(n=21)$ einen Schweregrad CEAP 4, 16,7\% ( $n=5)$ einen Schweregrad CEAP 5, 6,7\% ( $n=2)$ einen CEAP3 und 3,3\% $(n=1)$ einen Schweregrad CEAP 6. Die Venografie identifizierte die $\mathrm{V}$. femoralis als dominanten 
Zufluss bei 18 (60\%) und die V. femoralis profunda bei 12 (40\%) Patienten. Hinsichtlich Alter und Geschlechtsverteilung unterschieden sich diese beiden Gruppen nicht.

Als Prädiktoren für DFV als dominanten Zufluss wurden folgende Merkmale identifiziert: größerer DFV-Durchmesser $50 \mathrm{~mm}(8,73 \pm 4,34 \mathrm{~mm}$ vs. $11,9 \pm$ $3,52 \mathrm{~mm} ; \mathrm{p}=0,043)$ und $250 \mathrm{~mm}$ unterhalb des Trochanter minor $(5,4 \pm 3,90 \mathrm{~mm}$ vs.
$8,90 \pm 2,70 \mathrm{~mm} ; \mathrm{p}=0,011)$. Zweitens ein niedrigeres FV/DFV-Verhältnis $150 \mathrm{~mm}$ unterhalb dem Trochanter minor (11,39 $20,01 \mathrm{~mm}$ vs. $1,05 \pm 0,47 \mathrm{~mm} ; \mathrm{p}=0,043$ ) sowie drittens Vorhandensein von FV-Narben/Synechien $(p=0,003)$, Kollateralen $(p=0,003)$ und abnormaler Wanddicke $(p<0,0001)$. Das Fehlen und eine Duplikation der femoralen Vene, genauso wie ein Obturator-Zeichen oder pelvine Kollateralen erreichten keine statistische Signifikanz als Prädiktoren der DFV-Dominanz.

\section{FAZIT}

Die Ergebnisse dieser Studie deuten darauf hin, dass die direkte CT-Venografie bei der Bestimmung des dominanten Zuflusses zur Iliakalvene von Nutzen ist. Zur Validierung dieser vorläufigen Daten sind jedoch weitere Untersuchungen notwendig. Möglicherweise, so die beiden Autoren der Studie, kommen Zentren mit Expertise in der MRVenografie zu ähnlichen Ergebnissen.

Richard Kessing, Zeiskam 